\title{
FACTORES PROTECTORES E DE VULNERABILIDADE NA ADAPTAÇÃO EMOCIONAL E ACADÉMICA DOS FILHOS AO DIVÓRCIO DOS PAIS
}

\author{
Tatiana Carvalho Homem ${ }^{1}$ \\ Maria Cristina Canavarro ${ }^{2}$ \\ Ana Isabel Leite de Freitas Pereira ${ }^{3}$
}

Resumo: Até à década de 70, a separação/divórcio conjugal era preponderantemente analisada do ponto de vista moral e jurídico e conceptualizada como um acontecimento com consequências muito negativas para o desenvolvimento dos filhos do casal. Desde essa altura, progressivamente, abordagens menos lineares e disciplinarmente mais integradoras, têm oferecido uma perspectiva sobre o divórcio como um processo complexo, que envolve várias componentes (psicológicas, legais, económicas, parentais e sociais) e que exige adaptações familiares e individuais diversas, aos pais e aos filhos. Esta nova perspectiva implica uma atenção à diversidade de trajectórias desenvolvimentais e um foco nos mecanismos e processos responsáveis por essas diferenças. Este trabalho tem como tarefa caracterizar a adaptação (avaliada através do ajustamento emocional e académico) de crianças filhas de pais divorciados, comparando-a com a apresentada por crianças pertencentes a famílias intactas. A amostra da investigação, constituída por 426 crianças do $3^{\circ}$ e $4^{\circ}$ ano do $1^{\circ}$ ciclo, os seus pais e professores, foi recolhida em várias escolas públicas e privadas das regiões Norte, Centro e Sul de Portugal.

Palavras-chave: divórcio, ajustamento emocional, ajustamento académico, desenvolvimento.

\footnotetext{
${ }^{1}$ Unidade de Psicologia do Departamento de Psiquiatria e Saúde Mental do Hospital Infante Pedro, Aveiro.

${ }^{2}$ Faculdade de Psicologia e Ciências da Educação, Unidade de Intervenção Psicológica (UnIP). Universidade de Coimbra.

Maternidade Dr. Daniel de Matos - HUC, Coimbra.

${ }^{3}$ Escola Superior de Educação João de Deus, Lisboa.

Este projecto foi aprovado parcialmente pela Fundação Calouste Gulbenkian $\operatorname{Ref}^{\mathrm{a}} 56575$ - e pela linha de investigação - MEDVOC - do Instituto de Psicologia Cognitiva e Desenvolvimento Vocacional e Social, Unidade de I\&D - FEDER/POCTI-SFA$-160-192$.
} 
Risk and resilience factors in children's academic and emotional adjustment to their parents' divorce (Abstract): Until the 70's separation/divorce was mainly analysed from a moral and legal point of view and it was conceptualized as a situation with very negative consequences for children. Since then, less linear and more integrative points of view have been offering a new perspective on divorce, conceptualizing it as a complex process which includes many components (psychological, legal, economic, parental and social) and requires family and individual adaptations, both to parents and to children. This new perspective requires attention to the diversity of developmental trajectories and a new focus on the mechanisms and processes responsible for this diversity. The aim of this study is to characterize the adaptation (measured by the emotional and academic adjustment) of children from divorced families, compared to the adaptation of children from intact families. Our sample includes 426 children from third and fourth grades, their parents and teachers and it was collected in public and private schools in the North, Centre and South of Portugal.

Key-Words: divorce, emotional adjustment, academic adjustment, child development

\section{Introdução}

Desde o início do século XX que a estabilidade do casamento tem vindo a decrescer nas sociedades ocidentais. Portugal, a partir de 1974, tem acompanhado os outros países no aumento do número de rupturas conjugais e no ano de 2002, segundo dados do INE, por cada 100 casamentos celebrados ocorreram 49 divórcios decretados, com um acentuado predomínio dos divórcios por mútuo consentimento (cerca de $90.9 \%$ ).

Este aumento da taxa de divórcios parece estar relacionado com o aparecimento de diversos factores, entre os quais destacamos: (a) a entrada da mulher no mundo do trabalho, durante e após a segunda grande guerra, tornando possível a sua independência económica (Pinto \& Pereira, 2005); (b) o aparecimento de contraceptivos orais nos anos 60 , que veio permitir delinear novas estratégias de fecundidade das famílias; (c) a emergência de valores "individualistas", onde é dado o privilégio à procura da auto-realização em detrimento do compromisso com a família e com a instituição casamento (Pryor \& Rogers, 2001; Torres, 1996); (d) e, finalmente, o facto de, actualmente, o divórcio já não ser percepcionado pela sociedade como um acto marginal e culpabilizante. De facto, na Europa, o Catolicismo Romano impôs, durante muito tempo, doutrinas de indissolubilidade do casamento e em muitos países da Europa o divórcio civil só passou a ser 
possível já no século XX (em Itália em 1970; Portugal em 1910; Espanha apenas em 1981, Torres, 1996).

Estas modificações levaram a uma mudança profunda ao nível dos papéis e responsabilidades do homem e da mulher, da comunicação entre o casal e do poder relativo à tomada de decisão dentro da família. As relações conjugais tornaram-se menos estáveis do que anteriormente, tornando-se comum para muitas crianças viver o período de transição correspondente à separação e/ou divórcio dos pais, situação que requer uma adaptação a novas realidades familiares. Embora cada vez mais frequentes, estas modificações na estrutura familiar não são, normalmente, previstas (nem desejadas) pelas crianças e acabam por conduzir à necessidade de reorganização a diferentes níveis (familiar, económico e, fundamentalmente, de relações interpessoais).

No entanto, e apesar da frequência de divórcios nas sociedades actuais, só a partir dos anos 70 é que a separação conjugal passou a ser considerada como um processo complexo de transições envolvendo várias componentes - emocionais, legais, económicas, parentais e sociais - que pode comportar diversos desfechos desenvolvimentais e não como um acontecimento inequivocamente associado a consequências negativas (Amato, 2001; Emery, 1988).

Inicialmente, o divórcio foi conceptualizado como um desvio à normalidade e a investigação (Blechman, 1982; Levitin, 1979) tentava relacionar esta estrutura familiar menos desejável com consequências negativas para o ajustamento das crianças e para o seu desenvolvimento psicológico. Este modelo patogénico conduziu ao estudo de amostras clínicas durante o período de crise que se segue à situação de divórcio.

À medida que começaram a ser utilizadas amostras não clínicas, os investigadores foram descobrindo que, embora a maioria das crianças experiencie um período de crise quando os pais se separam, a longo-prazo os efeitos do divórcio dos pais são diferentes de criança para criança (Hetherington, Cox \& Cox, 1982). Estes resultados conduziram ao abandono do modelo patogénico e as consequências da separação parental passaram a ser enquadradas de acordo com modelos de stress e com modelos desenvolvimentais.

Wallerstein e Blackeslee (1989) perspectivam a separação parental como um poderoso factor de stress social que exige às crianças que lidem com determinadas tarefas para as quais não estão, muitas vezes, preparadas. Completar estas tarefas com sucesso, levando as crianças a não se desviarem de uma trajectória desenvolvimental adaptativa depende das estratégias de coping das crianças e do suporte social que lhes é disponibilizado para lidar com estes factores de stress.

Assim, tem-se vindo a salientar a importância de conceptualizar o divórcio, não como um acontecimento discreto, mas antes como mais um 
elo na cadeia de transições familiares, que deve ser analisado a diferentes níveis. A resposta a estas transições/crises familiares depende sempre daquilo que as precede e do que sucede posteriormente (Furstenberg \& Kiernan, 2001).

O divórcio representa uma transição prolongada nas vidas de pais e filhos e, para estes últimos, a situação de divórcio pode tornar-se particularmente difícil devido às capacidades parentais dos progenitores se encontrarem habitualmente diminuídas em muitas das suas dimensões (disciplina, tempo de brincar, apoio emocional).

Nos últimos anos, a investigação nesta área (Ahrons, 2007; Amato \& Keith, 1991; Cowan \& Cowan, 1990; Heatherington, 1981; Wallerstein \& Kelly, 1980) tem sido muito profícua e dela resulta a ideia consensual segundo a qual a maior parte das crianças filhas de pais separados/divorciados passa por uma fase inicial em que a separação parental é considerada como um factor causador de grande stress.

Alguns estudos (Ahrons, 2007; Edwards, 2002; Heatherington, Cox \& Cox, 1982) consideram que o divórcio representa uma perturbação à qual a maior parte dos indivíduos acaba por se adaptar com o tempo. Após um período de perturbação emocional e de crise, que segundo alguns autores teria a duração aproximada de três anos (Wallerstein \& Kelly, 1980), os filhos acabariam por voltar ao seu nível de funcionamento pré-divórcio.

No entanto, autores como Emery (1982) apontam numa direcção oposta, considerando que estar divorciado implica pressões persistentes, tais como as dificuldades económicas ou a solidão. Assim, o bem-estar psicológico das crianças não aumenta à medida que o tempo decorre após a situação de divórcio, ocorrendo antes o contrário. Diferentes investigadores (Heatherington, Stanley-Hagan \& Anderson, 1989; Wallestein \& Kelly, 1980) têm vindo a sugerir que as crianças filhas de pais divorciados apresentam níveis de perturbação mais elevados do que as crianças filhas de famílias intactas. Os conflitos conjugais e/ou a consequente separação parecem estar associados à emergência, a longo-prazo, de situações de depressão, desinvestimento, competência social baixa, problemas de saúde e baixo rendimento académico dos filhos (Amato \& Keith, 1991; Cowan \& Cowan, 1990; Emery \& O’Leary, 1982).

De acordo com as investigações que acabámos de referir, as consequências do divórcio para o sistema familiar não podem ser abordadas de forma simples e linear. Pelo contrário, elas são influenciadas por uma multiplicidade de factores que determinam a resposta da família a esta situação. A presença de factores de stress como o conflito, a perda, a mudança e a incerteza, presentes no período durante e imediatamente após o divórcio, leva a que os vários membros da família estejam sujeitos a diversas modificações nas suas vidas (Heatherington et al., 1982). 
De facto, têm sido propostas inúmeras explicações para justificar a forma como o divórcio poderá ter consequências negativas na vida das crianças e alterar as suas trajectórias desenvolvimentais.

De acordo com Amato e Keith (1991), as abordagens compreensivas sobre os efeitos a curto-prazo do divórcio dos pais na vida das crianças são habitualmente orientadas por um de três vectores: (1) ausência parental; (2) desvantagem económica e (3) conflito familiar.

Apresentamos em seguida, de forma sumária, cada um destes vectores:

\section{Ausência Parental}

A abordagem centrada na ausência parental atribui os presumíveis efeitos negativos do divórcio a défices no processo de socialização, resultantes do facto das crianças crescerem acompanhadas preponderantemente por um dos progenitores (Easterbrooks \& Emde, 1998; Emery, 1982). Baseia-se num pressuposto muito linear: se os progenitores são recursos muito importantes para o desenvolvimento das crianças, então, a presença dos dois progenitores será melhor que a de um só.

Esta perspectiva tem vindo a ser criticada pelo facto de colocar a ênfase na estrutura familiar, desvalorizando os processos familiares e por ser politicamente conservadora.

Vários estudos (Amato, 1987; Heatherington et al., 1982) mostram que o divórcio está associado a um decréscimo na quantidade e qualidade do contacto entre as crianças e os progenitores. Por razões óbvias, este facto verifica-se em relação ao progenitor que não fica com a sua custódia. Para além disto, como habitualmente o progenitor que detém a custódia das crianças tem de trabalhar, também ele tem constrangimentos quanto ao tempo e energia que pode dedicar aos filhos. Por estas razões, as crianças filhas de pais divorciados passam muitas vezes pela experiência de terem menos atenção, carinho e disponibilidade por parte dos seus pais. Este decréscimo no apoio parental pode aumentar a probabilidade de aparecerem determinados problemas como baixo rendimento académico, baixa auto-estima e problemas de comportamento. Por outro lado, a falta de modelos parentais no lar pode resultar numa aprendizagem inadequada das competências sociais necessárias para ter sucesso no mundo exterior.

\section{Desvantagem Económica}

A valorização deste vector, considera que o divórcio leva, habitualmente, a um declínio nos padrões de vida das mulheres/mães, muitas vezes levando-as mesmo a viver na pobreza. Assim, seriam as dificuldades económicas as principais responsáveis pelo mal-estar nas crianças (Demo, Fine \& Ganong, 2000). 
Esta falta de recursos económicos aumenta o risco de aparecimento de determinados problemas nas crianças (por exemplo, nas áreas de nutrição e saúde). Para além disto, as mães sozinhas, frequentemente, não têm capacidade económica que lhes permita pagar explicações, comprar brinquedos educativos, computadores e outro tipo de materiais que facilitam o sucesso académico das crianças. Os recursos limitados levam ainda a que, muitas vezes, as crianças tenham de viver em bairros problemáticos e com poucos recursos disponíveis. Nestes bairros as crianças têm menos acompanhamento e são menos monitorizadas por parte dos adultos, estando mais expostas à influência de grupos de pares marginais.

Deste vector decorrem diferentes hipóteses. Em primeiro lugar, que o bem-estar da criança aumenta se a mãe se casar de novo, já que esta união melhoraria as suas condições financeiras. No entanto, vários estudos revistos numa meta-análise de Amato e Keith (1991) não confirmam esta hipótese. Em segundo lugar, que as crianças terão menos problemas se viverem com o pai, já que este, habitualmente, ganha mais do que a mãe. Também aqui, várias investigações (Dunlop \& Burns, 1988; Gibson, 1969; Gregory, 1965; Peterson \& Zill, 1986; Steinhausen et al., 1987; Stephens \& Day, 1979, cit. por Amato \& Keith, 1991) sugerem que os rapazes que ficam a viver com as mães revelam um pior ajustamento psicológico, por comparação com as raparigas, enquanto que as raparigas que ficavam a viver com os pais apresentam um pior ajustamento, por comparação com os rapazes. Se os efeitos da custódia variam de acordo com o género das crianças e dos progenitores (comparação também realizada por Câmara \& Resnick, 1988), então este padrão parece apoiar mais uma perspectiva baseada na ausência parental do que a da desvantagem económica.

\section{Conflito Familiar}

A ênfase colocada neste vector salienta que o conflito conjugal antes e durante o período de separação é o principal factor de stress para as crianças. A hostilidade inter parental cria um ambiente familiar de tensão, no qual as crianças experienciam stress, infelicidade e insegurança.

De acordo com Easterbrooks e Emde (1988) diferentes mecanismos podem explicar os resultados negativos do conflito no casal. Por um lado, as crianças podem assumir os sintomas do conflito em si próprios, isto é, receando o divórcio dos pais, as crianças podem exibir mais comportamentos desadequados, numa tentativa de distrair os pais dos seus conflitos conjugais. Por outro lado, ao serem expostas a situações de conflito conjugal, as crianças aprendem por modelagem padrões negativos de resolução de conflitos, e com maior probabilidade utilizam estes mesmos padrões na gestão dos seus próprios conflitos (Easterbrooks \& Emde, 1988; Emery, 1982). 
Heatherington et al., (1982), formularam ainda uma hipótese de socialização, sugerindo que os conflitos conjugais podem também levar à inconsistência da disciplina dada aos filhos, que por sua vez teria consequências negativas para a adaptação dos filhos.

É também possível que certas características como a hostilidade ou o desinvestimento na relação conjugal possam tornar as interacções particularmente perturbadoras para as crianças. A frequência e a intensidade do conflito, bem como o grau em que as crianças são expostas directamente a estes conflitos, têm sido identificadas como dimensões com um grande impacto no desenvolvimento infantil (Heatherington et al.,1982).

De salientar que a hipótese do conflito familiar como principal factor de stress é aquela que tem recolhido maior suporte empírico (Easterbrooks e Emde, 1988; Heatherington et al.,1982; Pruett et al., 2003).

Ainda relativamente aos efeitos do divórcio dos pais a curto-prazo na vida das crianças, consideramos pertinente referir também a importância de uma quarta variável, a qualidade da relação entre pais e filhos.

A relação emocional ou de vinculação entre os progenitores e as crianças pode ser perturbada pelo conflito parental, havendo uma tendência para as crianças que vivem em lares mais conflituosos desenvolverem um estilo de vinculação mais inseguro (Cummings, Davies \& Campbell, 2000). Também neste sentido apontam diferentes investigações (por exemplo, Edwards, 2002; Frosch \& Mangelsdor, 2001) que consideram que a relação conjugal influencia de forma significativa o comportamento parental e os seus resultados ao nível do desenvolvimento das crianças, referindo que o conflito conjugal está associado a relações de vinculação inseguras.

Assim, o conflito conjugal e o stress parecem afectar o funcionamento da criança através do impacto negativo que têm sobre o comportamento parental. De um modo mais específico, a interacção entre o comportamento conjugal e o comportamento parental é muito importante para compreendermos a associação entre comportamento conjugal, comportamento parental e problemas de comportamento nas crianças, isto é, a combinação entre uma relação conjugal menos positiva e uma parentalidade menos apoiante e carinhosa, parece ser verdadeiramente problemática para as crianças (Frosch \& Mangelsdor, 2001).

Em síntese, inicialmente, o estudo dos efeitos do divórcio na família foi perspectivado em torno da emergência de problemas de comportamento após a separação. No entanto, investigações recentes (Edwards, 2002; Furstenberg \& Kiernan, 2001) têm colocado a ênfase num variado leque de respostas às transições de vida. Embora ainda se debata hoje a magnitude e duração dos efeitos da separação parental nas crianças, as investigações 
realizadas nos últimos dez anos têm concluído que, para compreendermos a variedade de respostas à situação de divórcio parental, temos de analisar as interacções entre as diferenças individuais e familiares e características extra-familiares.

Este reconhecimento de que o divórcio faz parte de uma cadeia de transições familiares e de experiências de vida e que a resposta individual a esta situação é muito variada, tem tido uma grande influência sobre os modelos teóricos que procuram explicar a forma como as crianças se adaptam à situação de separação parental (Furstenberg \& Kiernan, 2001).

Assim, ao abordarmos as consequências do divórcio para o desenvolvimento infantil a longo prazo, torna-se mais útil a utilização de uma grelha de leitura desenvolvimentista, como a que é oferecida pela Psicopatologia do Desenvolvimento, que considera que os factores que antecedem e precedem a transição familiar interagem com mudanças estruturais como o divórcio ou a formação de uma nova família, conceptualizando cada consequência como o resultado de um processo dinâmico que envolve diferentes componentes.

Uma abordagem desenvolvimentista permite-nos uma compreensão mais clara das implicações do divórcio para o desenvolvimento infantil e permite ainda, de uma forma mais precisa, o conhecimento dos factores protectores e dos factores de risco que podem promover ou dificultar um bom ajustamento emocional e académico à situação de divórcio parental.

Neste contexto, a presente investigação, realizada com crianças em idade escolar, tem como principal objectivo caracterizar e comparar sócio-demograficamente crianças de famílias divorciadas e crianças de famílias intactas, bem como comparar estes dois grupos em relação ao seu nível de ajustamento emocional e académico.

O presente estudo, tem ainda como objectivo analisar os factores sexo e tempo de divórcio enquanto possíveis moderadores do efeito do divórcio no ajustamento emocional e académico da criança. Em relação à primeira variável, o tempo decorrido desde o divórcio, vários estudos sugerem que após uma fase inicial de perturbação, a maioria das crianças atinge os níveis de funcionamento pré-divórcio (Ahrons, 2007; Edwards, 2002; Heatherington et al., 1982).

Quanto à variável sexo da criança, alguns estudos sugerem que os conflitos conjugais e a separação parental estão associados a problemas de controlo comportamental nos rapazes, enquanto que as raparigas parecem apresentar mais problemas de depressão e ansiedade (Amato \& Keith, 1991; Cowan \& Cowan, 1990; Emery \& O'Leary, 1982). Assim, o presente estudo, avaliando o ajustamento emocional através da consideração simultânea das duas principais dimensões de psicopatologia na infância, problemas de interiorização e problemas de externalização, torna possível a averiguação do impacto diferencial do divórcio em função do sexo das crianças. 


\section{Método}

A amostra total é constituída por 426 crianças que frequentavam o $1^{\circ}$ ciclo do ensino básico. As crianças tinham idades compreendidas entre os 8 e os 10 anos, sendo a idade média de 8,95. O grupo de crianças apresenta uma proporção equilibrada de crianças do sexo feminino e do sexo masculino $(52,5 \%$ de crianças do sexo masculino).

Foram constituídos dois grupos de participantes designados grupo de crianças filhas de pais divorciados, constituído por 67 crianças (presença de separação/divórcio parental) e grupo de comparação, constituído por 362 crianças (pertença a famílias intactas).

A análise comparativa dos grupos revelou que, relativamente ao sexo, ao nível de escolaridade, à idade das crianças e à idade dos pais das crianças não existem diferenças estatisticamente significativas entre os dois grupos. Também em relação ao nível sócio-económico não há diferenças significativas entre os grupos.

Foram encontradas diferenças estatisticamente significativas quanto à idade da mãe $(t=3,372, p<0,001)$ e ao número de irmãos $\left(\chi^{2}=15,62\right.$ $p<0,001)$.

Quanto ao grupo de crianças filhas de pais divorciados, salientamos que cerca de $31,3 \%$ das mães e $32,8 \%$ dos pais destas crianças voltaram a casar. No que diz respeito ao tempo de duração do divórcio dos progenitores, há uma maior percentagem de crianças filhas de pais divorciados há mais de 3 anos $(54,8 \%)$.

Importa salientar que a categorização desta variável em apenas duas categorias (divórcio há três ou menos anos e divórcio há mais de três anos) teve por base a literatura na área (por exemplo, Wallerstein \& Kelly, 1980) onde é referido que os três anos pós-divórcio correspondem ao período de maior crise na adaptação ao divórcio.

\section{Procedimentos}

Depois de concedida autorização pelas Direcções Regionais de Educação do Norte, Centro e Sul, os dados foram recolhidos em Escolas da Associação de Jardins Escolas João de Deus e em Escolas da Rede Pública, nas seguintes zonas geográficas: Norte, Centro, Lisboa e Vale do Tejo, entre Março de 2003 e Julho de 2003. A maior percentagem de crianças é oriunda da zona de Lisboa e da zona do Porto.

$\mathrm{O}$ facto da recolha da amostra ter sido efectuada com crianças de diferentes zonas do país teve como objectivo analisar a influência da pertença a diferentes zonas geográficas, com valores culturais distintos, na adaptação ao divórcio dos pais. 
Foi utilizado um protocolo constituído por diversos questionários para ser preenchido pelas crianças, pelos pais e pelos professores. O presente estudo recorreu apenas a alguns instrumentos que faziam parte de um protocolo de avaliação mais alargado realizado no âmbito do Projecto de Investigação: Dimensões Relacionais da Infância e Ajustamento Emocional e Académico Um estudo longitudinal com crianças do $1^{\circ}$ e $2^{\circ}$ ciclos do ensino básico.

Para a selecção da amostra, foram contactados professores do $1^{\circ}$ ciclo que leccionavam turmas de alunos dos $3^{\circ}$ e $4^{\circ}$ anos de escolaridade, tendo cada professor seleccionado aleatoriamente entre 3 a 11 alunos. Em seguida, foi também pedida a autorização dos pais para a recolha de dados e a sua colaboração para o preenchimento dos questionários.

\section{Instrumentos}

A bateria de avaliação utilizada na presente investigação inclui diferentes tipos de instrumentos de avaliação. Para além do Questionário de Identificação da Criança e da Família, preenchido pelos professores de todas as crianças que participaram no estudo e cujos dados se destinam à caracterização da amostra em termos sócio-demográficos (idade, sexo, questões relativas à situação escolar da criança, composição do agregado familiar, habilitações literárias e situação profissional dos pais, situação conjugal dos pais), no presente estudo foram utilizados o Child Behaviour Checklist de Achenbach (CBCL) e o Teacher's Report Form (TRF), também desenvolvido por Achenbach, nas versões para a população portuguesa de Fonseca, Simões, Rebelo, Ferreira e Cardoso, respectivamente de 1994 e 1995.

O CBCL é um questionário que permite registar, de forma estandardizada, as competências e os problemas de comportamento da criança e do adolescente, com base na informação fornecida pelos pais ou seus substitutos. Inclui vinte perguntas relativas às competências do indivíduo em áreas como a participação em desportos, passatempos, trabalhos, interacções sociais, desempenho escolar (quantitativa e qualitativamente) e 120 itens (dois deles itens abertos) relativos a diferentes problemas de comportamento e/ou perturbações emocionais registados numa escala de 0 a 2 ('não é verdadeiro' até 'é muito verdadeiro').

No presente estudo são utilizados os resultados do questionário relativos à pontuação global e os resultados para cada uma de duas dimensões (problemas de comportamento exteriorizados e problemas internalizados).

O TRF é um questionário que permite registar de forma estandardizada as competências e os problemas de comportamento da criança e do adolescente, com base na informação fornecida pelos professores. Consta de uma primeira parte com itens relativos a dados demográficos, avaliações do rendimento escolar e avaliação do funcionamento geral em sala de aula e 120 itens (dois deles itens abertos), 25 dos quais especificamente escolares e os restantes iguais aos da versão correspondente para pais. Os itens são 
registados numa escala de 0 a 2 ('não é verdadeiro' até 'é muito verdadeiro').

No presente estudo são utilizados os resultados do questionário relativos à pontuação global, os resultados para cada uma de duas dimensões (problemas de comportamento exteriorizados e problemas internalizados) e o resultado relativamente à competência académica.

Os estudos psicométricos das versões portuguesas de ambos os questionários indicam níveis adequados de fiabilidade e de validade (Fonseca et al., 1994, Fonseca et al., 1995; Pereira, 2007).

Os resultados dos estudos (Fonseca et al., 1994; Fonseca et al., 1995) relativos à fiabilidade revelam índices de consistência interna elevados para as pontuações globais do CBCL $(\alpha=0,93)$ e para as pontuações globais do TRF $(\alpha=0,93)$. No mesmo sentido os estudos (Pereira, 2007), relativos à fiabilidade das dimensões de internalização e externalização, também apresentam níveis elevados de consistência interna $(\alpha=0,84$ para o CBCL internalização, $\alpha=0,88$ para o CBCL externalização, $\alpha=0,89$ para o TRF internalização e $\alpha=0,95$ para o TRF externalização).

\section{Procedimentos estatísticos}

Num primeiro momento, foram realizados testes Qui-Quadrado (com correcção de Yates no caso das tabelas de contingência $2 \times 2$ ), de forma a explorar a existência de diferenças entre os dois grupos, crianças filhas de pais divorciados e crianças de "famílias intactas", quanto às características sócio-demográficas.

Num segundo momento, foram analisadas as diferenças entre os dois grupos quanto aos diferentes indicadores de ajustamento. De forma a podermos recorrer à utilização de testes paramétricos, as seis variáveis relativas ao ajustamento emocional - TRF total, TRF internalização, TRF externalização, CBCL total, CBCL internalização, CBCL externalização - que não seguiam uma distribuição normal, foram sujeitas a transformações logarítmicas, com o objectivo de obter distribuições mais aproximadas à normal. De seguida recorremos a testes $t$ de Student para amostras independentes, de forma a explorarmos a existência de diferenças entre os dois grupos quanto ao ajustamento emocional e académico.

Por último, foram realizadas análises suplementares, análises de variância a dois factores, $(3 \times 2)$, considerando os efeitos das variáveis situação conjugal e tempo de divórcio (não divorciado, divorciado há menos de 3 anos e divorciado há mais de 3 anos) e sexo das crianças (masculino e feminino), uma vez que na literatura o tempo de divórcio e o sexo das crianças são referidos como influenciando o ajustamento infantil à situação de divórcio parental. Quando se verificaram efeitos estatisticamente significativos realizaram-se comparações múltiplas par a par recorrendo ao procedimento Tukey HSD, após ter sido verificado o pressuposto da igualdade de variâncias. 
Para o tratamento estatístico dos dados utilizou-se a versão 12,0 do programa SPSS (Statistical Package for the Social Sciences).

\section{Resultados}

\section{Características sócio-demográficas}

No Quadro 1, podemos analisar as diferenças entre o grupo de crianças de famílias divorciadas e o grupo de crianças de famílias intactas quanto aos dados sócio-demográficos. Como podemos observar, verificam-se apenas diferenças estatisticamente significativas entre os dois grupos quanto ao tipo de residência e número de irmãos. Os resultados revelam um maior número de crianças que são filhas únicas e de crianças residentes em zona urbana no grupo de crianças de famílias divorciadas por comparação ao grupo de crianças de famílias intactas.

Quadro 1. Características sócio-demográficas dos dois grupos da amostra: frequência absoluta, frequência relativa e resultados dos testes $\chi^{2}$

\begin{tabular}{lcccc}
\hline & $\begin{array}{c}\text { Famílias } \\
\text { Divorciadas } \\
\mathbf{n = 6 7}(\mathbf{\%})\end{array}$ & $\begin{array}{c}\text { Famílias } \\
\text { Intactas } \\
\mathbf{n = 5 1 4}(\mathbf{\%})\end{array}$ & $\chi^{\mathbf{2}}$ & $\boldsymbol{p}$ \\
\hline $\begin{array}{c}\text { Tipo de Residência } \\
\text { Rural }\end{array}$ & $4(6,0)$ & $99(19,3)$ & & \\
$\quad$ Semi-Urbana & $17(25,4)$ & $163(31,7)$ & 11,685 & $\boldsymbol{p}<\mathbf{0 , 0 5}$ \\
$\quad$ Urbana & $45(67)$ & $249(48,4)$ & & \\
Nível Sócio-Económico & $18(26,9)$ & $180(35)$ & 4,663 & \\
$\quad$ Baixo & $25(37,3)$ & $149(29)$ & & \\
$\quad$ Médio & $20(29,9)$ & $166(32,3)$ & & \\
$\quad$ Alto & & & & \\
Recasamento de um dos progeni- \\
tores $\quad$ & $21(31,3)$ & & & \\
$\quad$ Pai & $22(32,8)$ & & & \\
$\quad$ Mãe & & & & \\
Irmãos & $27(40,3)$ & $97(18,9)$ & 85,012 & $\boldsymbol{p}<\mathbf{0 , 0 1}$ \\
$\quad$ Não tem irmãos & $30(44,8)$ & $305(59,3)$ & & \\
$\quad$ Um irmão & $8(11,9)$ & $88(17,1)$ & & \\
$\quad$ Dois irmãos & $2(3,0)$ & $24(4,7)$ & & \\
$\quad$ Três ou mais irmãos &
\end{tabular}

Nota: A informação sobre a classificação rural, semi-urbana e urbana foi fornecida pelos professores das crianças. 


\section{Ajustamento emocional e académico}

Posteriormente, procedemos à comparação dos resultados obtidos pelas crianças pertencentes a famílias intactas com os resultados obtidos pelas crianças pertencentes a famílias separadas/divorciadas nos questionários de ajustamento emocional e académico (Teacher's Report Form e Child Behaviour Checklist).

Como podemos observar no Quadro 2, não se verificam diferenças estatisticamente significativas em nenhum dos indicadores de ajustamento emocional e académico, avaliados pelos pais e pelos professores

Quadro 2: Ajustamento emocional e académico dos dois grupos da amostra: média, desvio padrão e resultados dos testes t. Comparação de crianças pertencentes a Famílias Intactas vs. Famílias Separadas/Divorciadas (TRF e CBCL)

\begin{tabular}{lccccc}
\hline & \multicolumn{3}{c}{ Famílias Intactas } & \multicolumn{3}{c}{$\begin{array}{c}\text { Famílias Separa- } \\
\text { das/Divorciadas }\end{array}$} \\
\cline { 2 - 6 } & $\boldsymbol{M}$ & $\boldsymbol{D P}$ & $\boldsymbol{M}$ & $\boldsymbol{D P}$ & Teste $\boldsymbol{n}$ \\
\hline TRF Total & 16,28 & 17,64 & 20,35 & 20,55 & $\mathrm{~ns}$ \\
TRF Internalização & 5,46 & 6,31 & 7,02 & 6,94 & $\mathrm{~ns}$ \\
TRF Externalização & 4,98 & 8,39 & 5,97 & 8,38 & $\mathrm{~ns}$ \\
Competência Académica & 3,42 & 0,80 & 3,25 & 0,79 & $\mathrm{~ns}$ \\
CBCL Total & 26,21 & 16,42 & 28,67 & 20,85 & $\mathrm{~ns}$ \\
CBCL Internalização & 7,95 & 5,57 & 8,99 & 7,36 & $\mathrm{~ns}$ \\
CBCL Externalização & 7,81 & 6,22 & 8,0 & 6,46 & $\mathrm{~ns}$ \\
\hline
\end{tabular}

Nota: Teste $t$ para amostras independentes

Por último, foram realizadas análises suplementares e análises de variância a dois factores considerando os efeitos de interacção entre as variáveis sexo e situação conjugal/tempo de divórcio. 
Quadro 3. Ajustamento emocional e académico dos dois grupos da amostra considerando os factores situação relacional/tempo de divórcio

\begin{tabular}{|c|c|c|c|}
\hline & & Factores & $p$ \\
\hline \multirow{5}{*}{ 秃 } & Total & - & - \\
\hline & Internalização & - & - \\
\hline & & & $\mathrm{F}_{1,417}=5,54$ \\
\hline & Externalização & Efeito principal do factor sexo da criança & $p=0,002$ \\
\hline & Competência académica & - & - \\
\hline \multirow{4}{*}{$\underset{\circlearrowright}{\mathcal{U}}$} & Total & - & - \\
\hline & & Efeito principal do factor situação conjugal & $\mathrm{F}_{2,398}=4,71$ \\
\hline & Internalização & dos pais/tempo de duração do divórcio & $p=0,001$ \\
\hline & Externalização & - & - \\
\hline
\end{tabular}

De acordo com os resultados das análises apresentadas no Quadro 3, observam-se efeitos principais do factor sexo da criança para a sub-escala exteriorização do TRF, ou seja, os professores percepcionam os rapazes como exibindo mais problemas de comportamento do que as raparigas.

Observam-se igualmente efeitos principais do factor situação conjugal dos pais/tempo de duração do divórcio para a sub-escala internalização do CBCL. As análises de comparações múltiplas recorrendo ao teste Tuckey revelam que as crianças filhas de pais divorciados há três ou menos anos percepcionadas pelos pais como tendo mais problemas de internalização do que as crianças de famílias intactas ou crianças filhas de pais divorciados há mais de três anos. De notar que não encontrámos efeitos de interacção entre o sexo e o tempo de divórcio. 


\section{Discussão}

Até meados da década de 70, a maior parte da literatura conceptualizava as consequências da separação parental para o desenvolvimento infantil de uma forma negativa. Actualmente, vários autores caracterizam o divórcio como um processo complexo e que engloba uma multiplicidade de factores, focalizando-se nos diferentes mecanismos de protecção e de vulnerabilidade infantil.

O presente estudo enquadra-se assim numa perspectiva mais desenvolvimentista, procurando analisar os factores que antecedem e sucedem à situação de divórcio.

Os resultados da presente investigação revelam que, ao comparar uma amostra de crianças oriundas de famílias divorciadas com uma amostra de crianças oriundas de famílias intactas, há diferenças estatisticamente significativas no que toca ao tipo de residência e número de irmãos. Os resultados revelam um maior número de crianças filhas únicas no grupo de famílias divorciadas por comparação ao grupo de crianças de famílias intactas. Estes resultados vão no mesmo sentido dos resultados de outros estudos, que indicam que nos casais com mais filhos é menos provável a ocorrência de divórcio do que nos casais que não têm filhos (por exemplo, Waite, Haggstrom \& Kanouse, 1985). Uma hipótese que colocamos para explicar os dados da presente investigação é o facto do maior número de filhos nas famílias intactas ser indicativo de uma relação conjugal satisfatória ou mais estável, ou ainda, o facto de nas famílias com mais filhos a sobrecarga financeira poder ser um constrangimento ao divórcio.

Já em relação à zona de residência, os resultados da nossa investigação parecem corroborar a ideia que nas zonas urbanas, por toda uma cultura mais individualista, pela crescente urbanização e pelo papel profissional e económico que a mulher tem vindo a assumir (Pinto \& Pereira, 2005), a probabilidade de haver divórcios aumente.

No que diz respeito ao ajustamento emocional e académico, e ao contrário dos resultados de outras investigações (Amato \& Keith, 1991; Cowan \& Cowan, 1990; Emery, 1982; Emery \& O'Leary, 1982; Hetherington \& Stanley-Hagan,1999) que sugerem que as crianças filhas de pais divorciados apresentam níveis de perturbação mais elevados do que as crianças filhas de famílias intactas, os dados aqui apresentados parecem indicar que, para a amostra em estudo, os índices de ajustamento académico e emocional não se mostraram discriminadores dos dois grupos de crianças.

Uma explicação possível para estes dados é o facto de a maior parte das famílias do grupo de famílias separadas/divorciadas da nossa amostra $(54,8 \%)$ encontrar-se divorciada há mais de três anos, podendo ter já ultra- 
passado o período que na literatura é descrito como período de crise e encontrando-se, por isso, mais adaptada à situação de divórcio. De facto, este resultado é confirmado pelos resultados das análises que consideram a variável tempo do divórcio: é no grupo de famílias separadas há menos de três anos que se verificam mais problemas de internalização, avaliados pelos pais, dados que corroboram os resultados referidos por diferentes investigações (Emery, 1988; Long \& Forehand, 1987; Wallerstein \& Kelly, 1980) que salientam que os efeitos mais negativos do divórcio parental tendem a desaparecer nos dois/três anos após a sua ocorrência. Parece ser consensual que, durante este período de tempo, poderão emergir algumas alterações comportamentais e quebra no rendimento académico das crianças, sendo por isso considerado um período de crise. Após este período, o comportamento das crianças tende a estabilizar.

Os resultados do presente trabalho vão ao encontro do que acima referimos, já que as crianças cujos pais estão separados há três ou menos anos são percepcionadas pelos pais como revelando, globalmente, maiores dificuldades de ajustamento emocional. No entanto, importa salientar que estes resultados, para além de poderem representar diferenças "reais" no ajustamento deste grupo de crianças, podem dever-se também a diferenças na percepção dos progenitores. É possível que os pais destas crianças estejam mais atentos a possíveis alterações de comportamento e mais preocupados com o seu bem-estar psicológico, podendo mesmo sobrevalorizar alguns comportamentos das crianças e justificando-se assim as pontuações mais elevadas obtidas por este grupo no CBCL.

O sexo das crianças e o seu impacto no ajustamento emocional e académico após o divórcio parental, tem sido uma das variáveis mais estudadas nesta área (Amato \& Keith, 1991; Emery \& O’Leary, 1982; Heatherington \& Stanley-Hagan, 1999; Wallerstein \& Kelly, 1980), não sendo os resultados apresentados consensuais. Alguns autores (por exemplo, Amato \& Keith, 1991; Wallerstein \& Kelly, 1980) sugerem que o divórcio origina mais problemas de comportamento nos rapazes do que nas raparigas, sobretudo quando são mais jovens, enquanto que outros (por exemplo, Hetherington \& Stanley Hagan, 1999) salientam um aumento de problemas de comportamento nas raparigas adolescentes em relação aos rapazes adolescentes. A investigação tem mostrado também que os rapazes respondem à situação de separação parental com um aumento de problemas do comportamento exteriorizado, enquanto que as raparigas teriam mais problemas ao nível do comportamento internalizado (Emery \& O’Leary, 1982).

No presente estudo, os resultados não parecem corroborar os dados acima descritos, uma vez que não se verificaram efeitos moderadores da variável sexo, considerando as diferentes dimensões de ajustamento estudadas. 
No geral, estes resultados parecem apoiar a visão mais recente sobre as consequências da separação parental para o desenvolvimento infantil, visão esta que des-catastrofiza o carácter traumatizante deste acontecimento de vida, defendendo antes a existência de uma grande variabilidade nas respostas das crianças a esta situação, que irá depender da interacção entre as características individuais, familiares e extra-familiares da criança.

É importante salientar algumas limitações do nosso estudo, que colocam reservas à generalização dos resultados e às conclusões inferidas.

Uma primeira limitação está relacionada com os métodos de recolha de dados. Embora tenham sido utilizadas múltiplas fontes de informação (crianças, pais e professores), a utilização de questionários de auto-preenchimento como metodologia de avaliação apresenta algumas limitações decorrentes, por exemplo, de factores relacionados com a compreensão das escalas, com a motivação dos sujeitos e com possíveis enviesamentos. Ainda em termos metodológicos, o estudo não permite estabelecer a causalidade empírica das relações encontradas, sendo o seu sentido determinado através do quadro conceptual de referência.

Para além disto, as dimensões muito diferentes das amostras e o facto de, quando analisámos os efeitos do divórcio, não termos controlado factores importantes como o nível de conflito entre os progenitores, as outras transições pós-divórcio (por exemplo, o recasamento de um dos progenitores), o nível sócio-económico e a qualidade da relação entre pais e filhos, constituem também limitações do nosso estudo.

Apesar destas limitações, consideramos que o presente estudo tem alguns pontos fortes que julgamos importante salientar, nomeadamente, a dimensão da amostra, o recurso a múltiplos informadores para avaliar o ajustamento da criança, o facto de termos tido em consideração os diferentes domínios de ajustamento, incluindo as dimensões de internalização e de exteriorização.

Assim, torna-se importante passarmos, numa segunda fase do nosso estudo, à análise de algumas variáveis que poderão estar a funcionar como factores protectores para estas crianças, permitindo-lhes assim manifestarem comportamentos ajustados e bem-estar psicológico. Este objectivo vai ao encontro da perspectiva de vários autores (Heatherington et al.,1982; Wallerstein, 1989) que defendem que as consequências da separação parental para o desenvolvimento infantil dependem, sobretudo, das suas estratégias de coping e do suporte social que é disponibilizado para lidar com estes factores de stress. Para além disto, enquadra-se também nos objectivos das grelhas de leitura desenvolvimentistas, interessando-se pelas crianças filhas de pais divorciados enquanto grupo de risco (em termos de ajustamento emocional e académico) e tornando importante a compreensão de quais os mecanismos e processos que moderam as consequências dos factores de risco. 


\section{Referências}

Ahrons. C. (1983). Divorce: Before, during and after. In H. McCubbin \& C. Figley (Eds), Stress and the family: Coping with normative transitions (pp. 102-115). New York: Brunner/ Mazel Publishers.

Amato, P. (2001). Children of divorce in the 1990s: An update of the Amato and Keith (1991) meta-analysis. Journal of Family Psychology, 15, 355-370.

Amato, P., \& Keith, B. (1991). Parental divorce and the well being of children: A meta-analysis. Psychological Bulletin, 110 (1), 26-46.

Blechman, E. A. (1982). Are children with one parent at psychiatric risk? A methodological review. Jornal of Marriage and the Family, 44, 179-195.

Camara, K., \& Resnick, G. (1988). Interparental conflict and cooperation: Factors moderating children's post-divorce adjustment. In M. Hetherington \& J. Arasteh (Eds.), Impact of divorce, single parenting, and stepparenting on children (pp. 169-195). New Jersey: Erlbaum Publishers.

Cowan, P. A., \& Cowan, C. P. (1990). Becoming a family: Research and intervention. In I. Siegel \& G. Brody (Eds.), Family Research (pp. 1-51). Hillsdale, N. J.: Lawrence Erlbaum Associates.

Cummings, E., Davies, P., \& Campbell, S. (2000). Developmental psychopathology and family process: Theory, Research and clinical implications. New York: The Guilford Press.

Demo, D., Fine, M., \& Ganong, L. (2000). Divorce as a family stressor. In P. McKenry \& S. Price, (Eds.), Families \& Change: Coping with stressful events and transitions (pp. 279-302). Sage Publications.

Easterbrooks, M., \& Emde, R. (1998). Marital and parent-child relationships: The role of affect in the family system. In K. Hinde \& J. Stevenson-Hinde (Eds.), Relationships within families: Mutual influences (pp. 83-103). Oxford: University Press.

Edwards, M. (2002). Attachment, mastery and interdependence: A model of parenting processes. Family Process, 41(3), 389-404.

Emery, R. (1982). Interparental conflict and the children of discord and divorce. Psychological Bulletin, 92, 310-330.

Emery, R. (1988). Marriage, divorce and children's adjustment. USA: Sage Publications.

Emery, R. E., \& O’Leary, K. D. (1982). Children's peceptions of marital discord and behavipur problems of boys and girls. Journal of Abnormal Child Psychology, 10, 11-24.

Fonseca, A. C., Simões, A., Rebelo, J. A., Ferreira, J. A., \& Cardoso, F. (1994). Um Inventário de competências sociais e de problemas de comportamento em crianças e adolescentes: O Child Behaviour Checklist de Achenbach (CBCL). Psychologica, 12, 55-78.

Fonseca, A. C., Simões, A., Rebelo, J. A., Ferreira, J. A., \& Cardoso, F. (1995). O Inventário de comportamentos da criança para professores Teachers' Report Form (TRF). Revista Portuguesa de Pedagogia, 2, 81-102. 
Frosch, C., \& Mangelsdorf, S. (2001). Marital behaviour, parenting behaviour and multiple reports of preschoolers? Behaviour problems: Mediation or moderation?. Developmental Psychology, 37(4), 502-519.

Furstenberg, F., \& Kiernan, K. (2001). Delayed parental divorce: How much do children benefit?. Journal of Marriage and Family, 63(2), 446-458.

Hetherington, E. (1991). The role of individual differences and family relationships in children's coping with divorce an marriage. In P. Cowan \& M. Hetherington (Eds.), Family transitions (pp. 165-194). Lawrence Erlbaum Associates, Publishers.

Hetherington, E., Cox, M., \& Cox, R. (1982). Effects of divorce on parents and children. In M. Lamb (Ed.), Nontraditional families: Parenting and child development (pp. 233-287). Hillsdale, N. J.: Lawrence Erlbaum Associates.

Hetherington, E., \& Stanley-Hagan, M. (1999). The adjustment of children with divorced parents: A risk and resiliency perspective. Journal of Child Psychology and Psychiatry, 40, 129-140.

Hetherington, E., Stanley-Hagan, M., \& Anderson, E. (1989). Marital transitions: A child's perspective. American Psychologist, 44(2), 303-312.

Levitin, T. E. (1979). Children of Divorce. Journal of Social Issues, 35, 1-25.

Long, M., \& Forehand, R. (1987). The effects of parental divorce and parental conflict on children: An overview. Developmental and Behavioural Paediatrics, 8, 292-296.

Pinto, H., \& Pereira, M. (2005). Separação e divórcio: Um olhar no feminino. Coimbra: Quarteto Editora.

Pryor, J., \& Rodgers, B. (2001). Children in changing families: Life after parental separation. USA: Blackwell Publishers.

Pruett, M., Williams, T., Insabella, G., \& Little, T. (2003). Family and legal indicators of child adjustment to divorce among families with young children. Journal of Family Psychology, 17(2), 169-180.

Torres, A. (1996). Divórcio em Portugal: Ditos e interditos. Oeiras: Celta Editora.

Waite, L. J., Haggstroom, G.W., \& Kanouse, D. E. (1985). The consequences of parenthood for the marital stability of young adults. American Sociological Review, 50, 850-857.

Wallerstein, J., \& Blakeslee, S. (1989). Second chances. UK: Bantam Press.

Wallerstein, J., \& Kelly, J. (1980). Surviving the breakup. New York: Basic Books. 